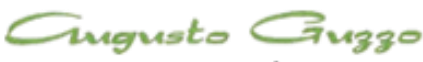

REVISTA ACADEMICA

\title{
Dimensionamento do sistema cremalheira-pinhão de dentes
}

\section{retos}

\author{
José Carlos Leite Lopes e Luciano Galdino²
}

Recebido em: 09/10/2013. Aprovado em: 10/11/2013. Disponibilizado em: 20/12/2013

1. Líder de Produção na Mecano Fabril Ltda. Tecnólogo em Mecatrônica Industrial pela Faculdade ENIAC. Graduando em Engenharia Mecatrônica na Faculdade ENIAC e Pós graduando em Engenharia da Manutenção Industrial na Faculdade ENIAC. E-mail: jcl_lopes@hotmail.com.

2. Professor de Física e Elementos de Máquina na Faculdade ENIAC. Mestre em Ciências Exatas e da Terra na área de Física Nuclear pela USP, especializado em Física pela USP e Licenciado em Matemática pela UNG. E-mail: lucianogaldino1@yahoo.com.br

\section{Resumo}

A cremalheira é um elemento de máquina muito importante para a execução de movimentos mecânicos, tanto horizontais como verticais, e esse artigo aborda de forma objetiva as etapas para o seu dimensionamento, demonstrando de maneira funcional os cálculos necessários para a execução do projeto desse fundamental elemento de transmissão mecânica. A cremalheira necessita que haja um pinhão acoplado a um motor, então se faz necessário também obter algumas características para o dimensionamento e fabricação do pinhão. Além de indicar as etapas passo a passo para o dimensionamento com suas respectivas equações, estão disponibilizados também os materiais adequados para fabricação da cremalheira e do pinhão de acordo com a sua aplicação, assim como o perfil de acabamento dos dentes de engrenamento.

Palavras-chave: Cremalheira. Pinhão. Dimensionamento. Materiais.

\begin{abstract}
The rack is a very important part of the machinery for the implementation of both horizontal and vertical mechanical movement, and this article discusses objectively the steps for your sizing, demonstrating a functional way the calculations necessary for the implementation of this fundamental design element mechanical transmission. The rack requires that there is a pinion coupled to an engine, so it is necessary to also get some features for the design and manufacture of the pinion. Besides indicating the steps walkethrough for sizing with their respective equations, are also available suitable materials for manufacturing rack and pinion according to their application, as well as the profile of finishing gear teeth.
\end{abstract}

Keywords: Rack. Pinion. Sizing. Materials 


\section{Introdução}

Devido à escassez de informações referentes ao dimensionamento de cremalheira e à importância que este elemento mecânico possui na indústria e também para fins acadêmicos, verificou-se a necessidade de elaborar um trabalho que direcione para um dimensionamento claro e objetivo.

A cremalheira é um dispositivo mecânico de formato reto que ajuda na realização de movimentos verticais e horizontais e permite o transporte das mais variadas cargas. $\mathrm{O}$ conjunto pinhão/cremalheira está associado a uma aplicação específica e isso demonstra que se devem conhecer as cargas e solicitações que o sistema de transmissão estará submetido a fim de se optar pelo melhor dimensionamento do elemento (SHIGLEY, 1984).

O pinhão/cremalheira é um sistema onde a coroa, que é a cremalheira, tem um diâmetro infinito, tornando-se reta. Os dentes podem ser retos ou inclinados. $\mathrm{O}$ dimensionamento é semelhante às engrenagens cilíndricas retas ou helicoidais, mas o problema é que a cremalheira é linear e não circular como as engrenagens citadas e isso faz com que alguns parâmetros sejam diferentes, Educação Tecnológica (s.d.).

Os passos necessários para fazer o dimensionamento são apresentados em detalhes nesse trabalho. São apontados os estudos das forças envolvidas nesse tipo de transmissão, tanto para o movimento horizontal como para o movimento vertical, através do uso de imagens ilustrativas e através da demonstração das equações necessárias, mas sempre apoiadas nas leis de Newton. São indicados também como devem ser as análises das velocidades e das acelerações através da movimentação pretendida para o projeto. Para determinação do módulo da cremalheira foi feito uso de gráficos do catálogo da empresa espanhola Koom (s.d.) da velocidade em função da força tangencial de acordo com o material que deve ser utilizado.

O dimensionamento do pinhão foi baseado no livro de elementos de máquina do autor Melconian (2008), mas utilizando como parâmetros iniciais os valores obtidos no dimensionamento da cremalheira.

O trabalho é concluído com explicações sobre o acabamento superficial, pois este é um fator muito importante para finalização da construção do conjunto pinhão/cremalheira (BUDYNAS, 2005, p. 642).

\section{Conhecendo o pinhão e a cremalheira}

O sistema pinhão/cremalheira é um dispositivo mecânico projetado para funcionar por longos períodos de trabalho e mantendo sua funcionalidade. Para realização de um projeto como esse são empregadas à ciência juntamente com a criatividade e arte da engenharia, Shigley (1984). O projeto é baseado na resistência dos materiais utilizados e também é considerado o desgaste que ocorre ao longo do tempo de uso. $\mathrm{O}$ pinhão pode ser projetado com dentes retos, quando a transmissão é feita entre eixos paralelos, Sardá (s.d.). Utilizando-se o modelo de dentes retos fica mais fácil fazer $o$ dimensionamento e também tem a vantagem de admitir grandes relações de transmissão, mas apresenta a desvantagem da geração de ruídos excessivos quando submetidos a altas rotações. A engrenagem cilíndrica de dentes helicoidais é apropriada para cargas e velocidades elevadas. A figura 1 ilustra o conjunto pinhão cremalheira. 
Figura 1: Representação do sistema pinhão e cremalheira

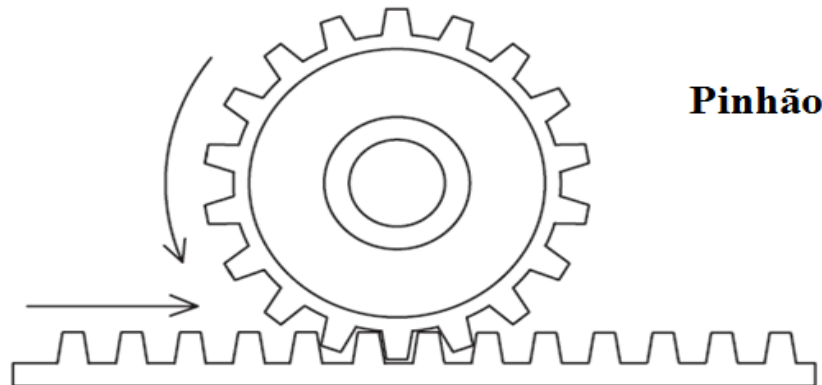

Cremalheira

Fonte: Transmissão e transformação de movimento (Clube da Eletrônica/Mecânica)

A equação1 apresentada a seguir deve ser utilizada para calcular o espaço percorrido pelo pinhão referente à cremalheira em uma rotação completa:

$\mathrm{e}=\pi \cdot \mathrm{d}$

Onde:

$\mathrm{e}=$ espaço percorrido em uma rotação do pinhão;

$\mathrm{d}=$ diâmetro primitivo do pinhão.

O diâmetro primitivo (d) é obtido através do produto entre o módulo do engrenamento (m) e o número de dentes $(Z)$, conforme equação 2.

$\mathrm{d}=\mathrm{m} \cdot \mathrm{Z}$

Substituindo a equação 2 na equação 1, obtém-se o espaço percorrido em uma volta (e) em função do módulo e do número de dentes (equação 3):

$\mathrm{e}=\pi \cdot \mathrm{m} \cdot \mathrm{Z}$

Para melhor compreensão do espaço percorrido em uma volta é apresentada a figura 2 a seguir:

Figura 2: Deslocamento da cremalheira ao fim de uma volta da roda (Pinhão).

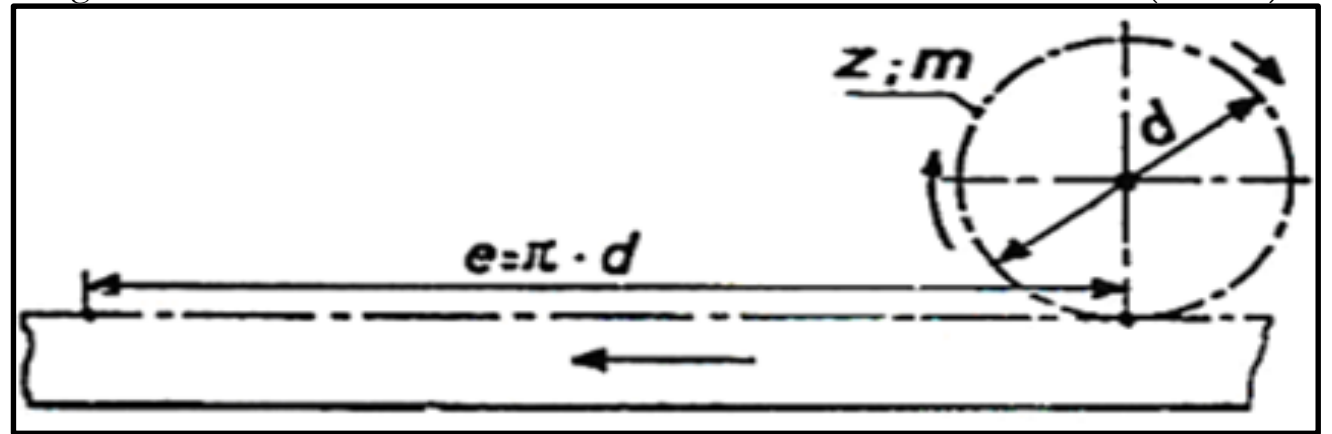

Fonte: http://elearning.iefp.pt

Os dados para verificação das distâncias de do pinhão/cremalheira, segundo o catálogo da KOOM (s.d.), permitem que o encaixe seja o 
melhor possível, pois é necessário respeitar a distância entre o eixo do pinhão e a base da cremalheira, sendo que essa dimensão depende do módulo, do diâmetro do eixo e se o pinhão é reto ou helicoidal, portanto existe uma distância correta específica para cada pinhão, conforme indicado na figura 3 .
A tabela 1 apresenta a relação entre a cota $\mathrm{D}$, o número de dentes do pinhão e o módulo do engrenamento, segundo o catálogo Koom, para pinhão com dentes retos.

Figura 3: Distância entre eixo e a base da cremalheira

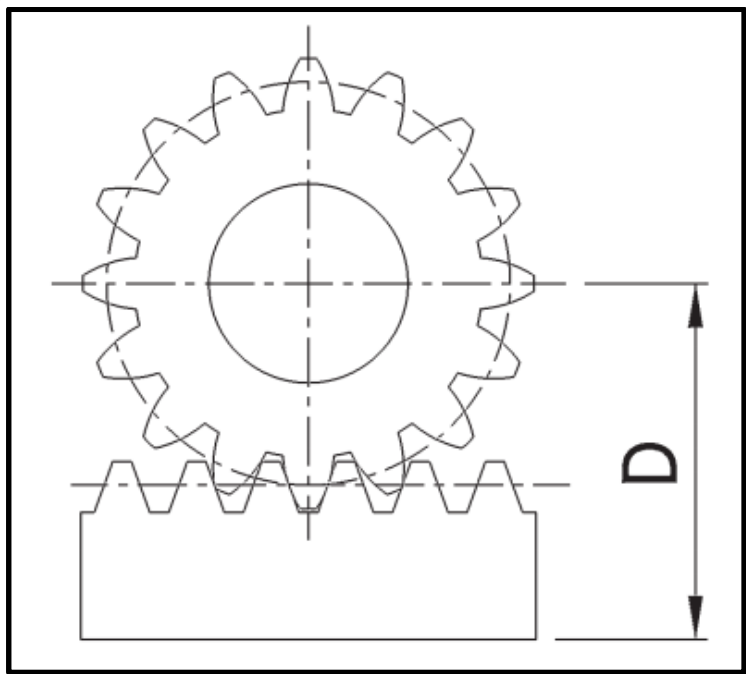

Fonte: Catálogo da Koom

Tabela 1: Escolha da distância entre eixo e base da cremalheira

\begin{tabular}{|c|c|c|c|c|c|c|c|}
\hline \multicolumn{7}{|c|}{ DIMENSÕES DA COTA “D” } \\
\hline & $\mathbf{7}$ & \multicolumn{7}{c|}{ Módulo do Pinhão com dentes retos (m) } \\
\cline { 2 - 8 } $\mathbf{Z}$ & $\mathbf{2}$ & $\mathbf{3}$ & $\mathbf{4}$ & $\mathbf{5}$ & $\mathbf{6}$ & $\mathbf{8}$ & $\mathbf{1 0}$ \\
\hline $\mathbf{1 8}$ & - & 62 & 81 & 104 & - & 157 & 195 \\
$\mathbf{2 0}$ & - & 65 & 85 & 109 & 125 & 165 & 205 \\
$\mathbf{2 2}$ & - & 68 & 89 & 114 & 131 & 173 & 215 \\
$\mathbf{2 4}$ & 50 & 71 & 93 & 119 & 137 & 181 & 225 \\
$\mathbf{2 6}$ & 52 & 74 & 97 & 124 & 143 & 189 & 235 \\
$\mathbf{2 8}$ & 54 & - & - & 129 & 149 & 197 & - \\
$\mathbf{3 0}$ & 56 & - & - & - & 155 & - & - \\
$\mathbf{3 2}$ & 58 & - & - & - & 161 & - & - \\
\hline
\end{tabular}

Fonte: Catálogo da Koom

A construção das cremalheiras se dá com diferentes tipos de materiais e a tabela 2 indica quais os materiais, segundo a norma espanhola UNE e o equivalente ABNT, podem ser utilizados, baseado no esforço que estará sujeita a cremalheira, juntamente com o tipo de tratamento térmico, assim como tolerâncias, precisão de perfil e rugosidade. 
Tabela 2: Tipo de materiais empregados na fabricação de cremalheiras.

\begin{tabular}{|c|c|c|c|c|c|c|c|c|}
\hline \multicolumn{9}{|c|}{ CAPACIDADES MECÂNICAS } \\
\hline \multirow[b]{2}{*}{ Material } & \multicolumn{2}{|c|}{ Tipo } & \multirow{2}{*}{$\begin{array}{l}\text { Tratamento } \\
\text { Térmico }\end{array}$} & \multirow[b]{2}{*}{ Capacidade } & \multirow{2}{*}{$\begin{array}{l}\text { Precisão do } \\
\text { perfil }\end{array}$} & \multirow[b]{2}{*}{$\mathrm{Ra}$} & \multicolumn{2}{|c|}{ Tolerâncias } \\
\hline & $\begin{array}{c}\text { Espanhol - } \\
\text { UNE }\end{array}$ & $\begin{array}{c}\text { Equivalente } \\
\text { ABNT }\end{array}$ & & & & & Passo & Total \\
\hline I & F-114 & $\mathrm{SAE}-1045$ & $\begin{array}{c}\text { Recozimento } \\
\text { isotérmico } \\
\text { com posterior } \\
\text { têmpera e } \\
\text { revenimento } \\
\end{array}$ & $>70 \mathrm{~kg} / \mathrm{mm}^{2}$ & $\begin{array}{c}\text { Classe 8 } \\
\text { segundo DIN } \\
3962\end{array}$ & $<0,8$ & 0,01 & 0,15 \\
\hline II & F-114 & $\mathrm{SAE}-1045$ & $\begin{array}{c}\text { Recozimento } \\
\text { isotérmico } \\
\text { com posterior } \\
\text { têmpera e } \\
\text { revenimento }\end{array}$ & $>52 H R C$ & $\begin{array}{c}\text { Classe 5 } \\
\text { segundo DIN } \\
3962\end{array}$ & $<0,8$ & 0,003 & 0,04 \\
\hline III & F-1540 & $\begin{array}{c}\mathrm{SAE}-4320 / \\
4320 \mathrm{H}\end{array}$ & $\begin{array}{c}\text { Cementado, } \\
\text { temperado e } \\
\text { revenido }\end{array}$ & $58-62 H R c$ & \begin{tabular}{|c|} 
Classe 5 \\
segundo DIN \\
3962
\end{tabular} & $<0,8$ & 0,003 & 0,04 \\
\hline
\end{tabular}

Fonte: Catálogo da Koom

\section{Dimensionamento da cremalheira de}

\section{dentes retos}

A função da cremalheira é de fazer a transformação de um movimento circular gerado por um motor e transmitido por um pinhão em movimento retilíneo ou viceversa, dependendo da necessidade.

A primeira etapa para o dimensionamento de uma cremalheira é determinar a velocidade linear máxima desejada para o projeto. Para isso, utiliza-se a equação 4 (HALLIDAY, 2012), onde deve-se estabelecer o espaço percorrido $(\Delta \mathrm{S})$ e o intervalo de tempo desejado $(\Delta \mathrm{t})$ para se completar esse espaço com a velocidade máxima (v).

$$
\mathrm{v}=\Delta \mathrm{S} / \Delta \mathrm{t}
$$

Após a obtenção da velocidade máxima, deve-se determinar a aceleração (a) do sistema para ele sair do repouso e atingir essa velocidade máxima, para isso, deve-se utilizar a equação 5 (HIBBELER, 2011). Novamente o projetista deverá avaliar qual o melhor intervalo de tempo $(\Delta \mathrm{t})$ para essa variação de velocidade $(\Delta \mathrm{v})$.

$$
\mathrm{a}=\Delta \mathrm{v} / \Delta \mathrm{t}
$$

O próximo passo é fazer um estudo das forças que estão envolvidas nessa transmissão. Devem ser consideradas duas opções inicialmente: a força tangencial horizontal (figura 4) e/ou a força tangencial vertical (figura 5). A única diferença para realização dos cálculos refere-se ao sentido da força que deve ser considerada para obtenção do movimento do sistema. 
Figura 4: Força tangencial horizontal

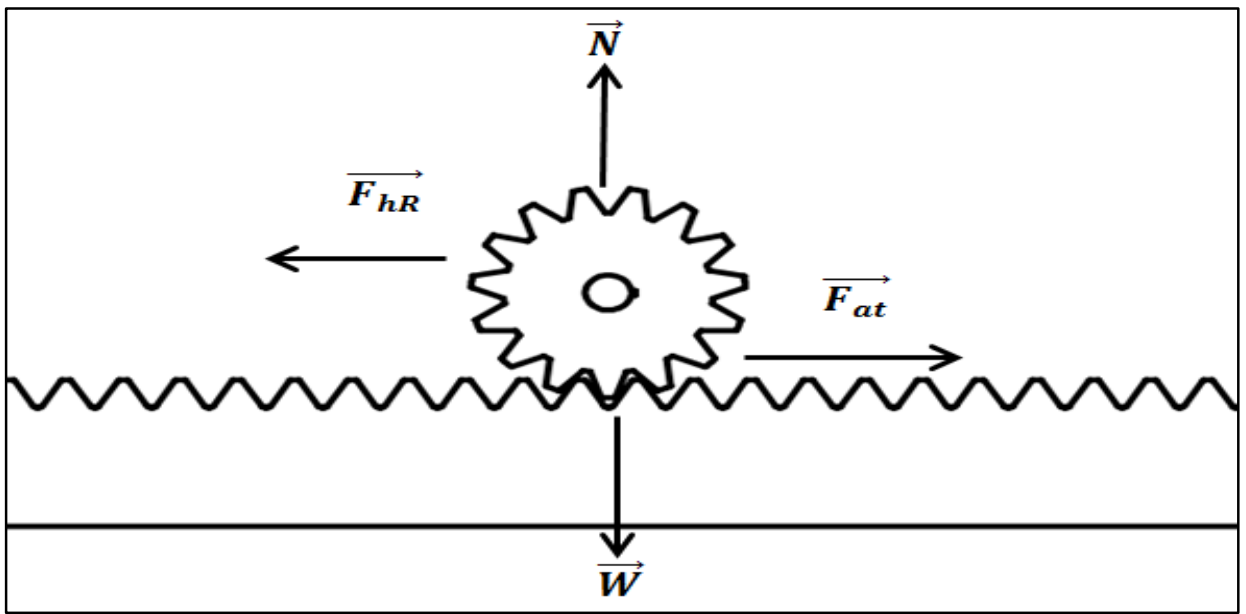

Fonte: Elaborado pelo autor no Solidworks

Figura 5: Força tangencial vertical

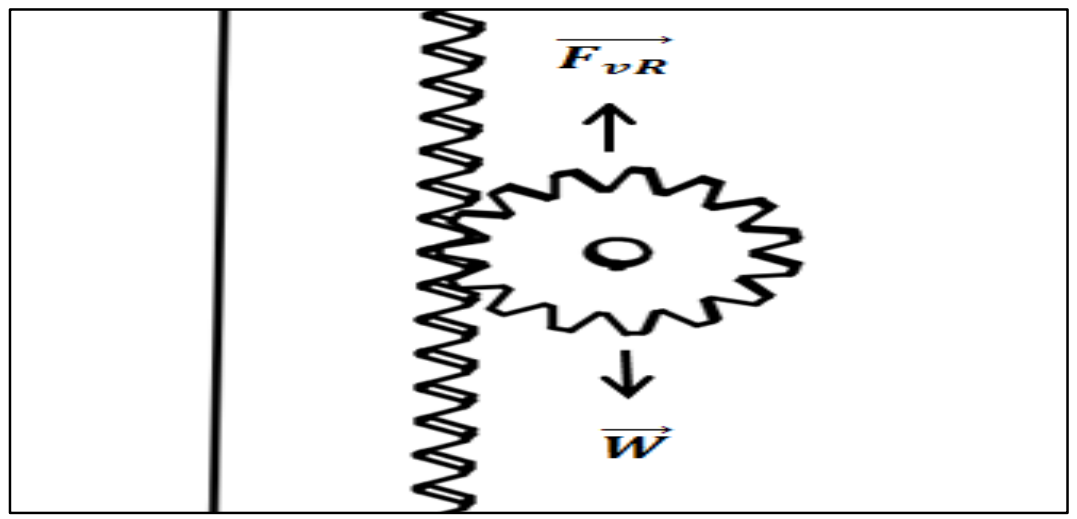

Fonte: Elaborado pelo autor no Solidworks

Existem várias aplicações que se utilizam do conjunto cremalheira/pinhão. Uma aplicação verificada para força tangencial horizontal está ilustrada na figura 6. Para o uso da força tangencial vertical foi encontrado a aplicação na empresa metalúrgica Mecano Fabril e refere-se a uma furadeira de bancada, como mostrado na figura 7 . 
Figura 6: Aplicação da força tangencial horizontal no sistema cremalheira/pinhão em acionamento de portão automático.

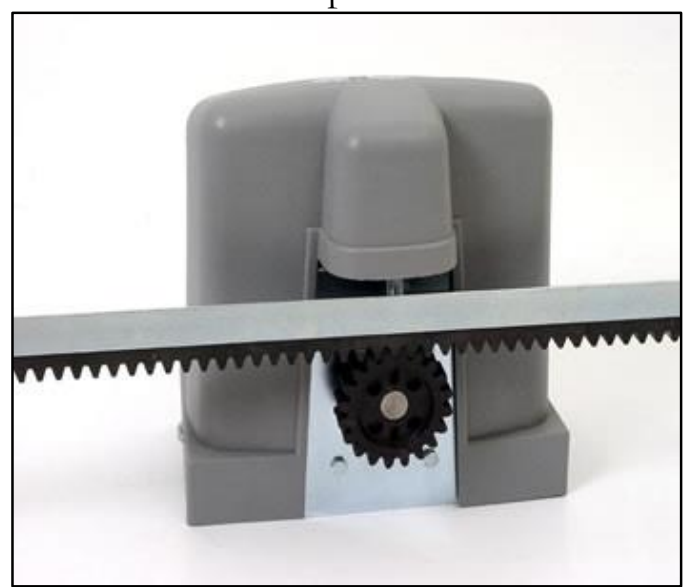

Fonte: RLG - Indústria e Equipamentos de Segurança Ltda.

Figura 7: Aplicação de cremalheira/pinhão com força vertical em furadeira de bancada

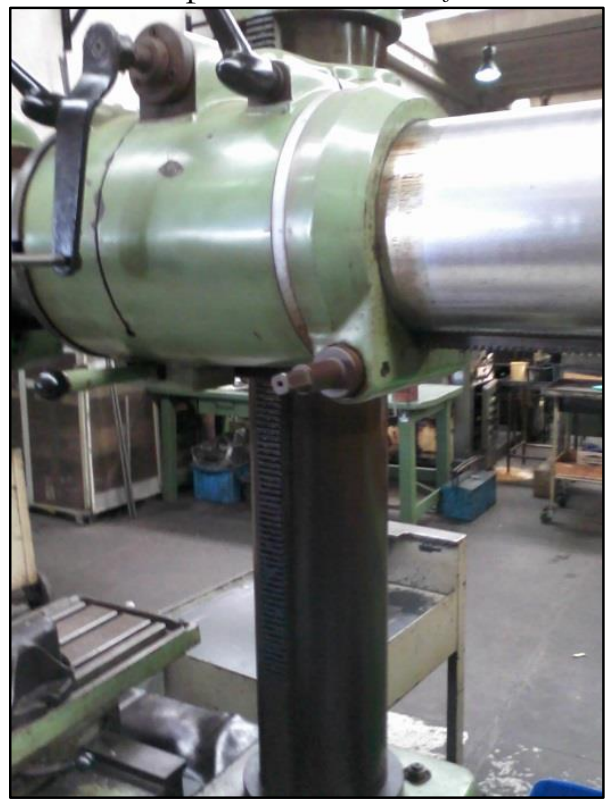

Fonte: Metalúrgica Mecano Fabril

A força tangencial horizontal real é definida a partir da segunda lei de Newton (equação 6) e depende da força de atrito (Fat), da força peso (W) e da força normal $(\mathrm{N})$, conforme figura 4 .

$$
\sum \vec{F}=m \cdot \vec{a}
$$

$$
\begin{aligned}
& \mathrm{m}=\text { massa a ser movida }(\mathrm{kg}) ; \\
& \mathrm{a}=\text { aceleração linear }\left(\llbracket \mathrm{m} / \mathrm{s} \rrbracket \wedge^{2}\right) ;
\end{aligned}
$$

Assim, substituindo as forças envolvidas na equação 6, têm-se:

$$
F_{h R}-F_{a t}=m \cdot a
$$

Onde: 
Como a força de atrito é dada por $\vec{F}_{a t}=$ $\mu . \vec{N}$, sendo a força normal igual à força peso $(N=W)$ e sendo a força peso dada por $\vec{W}=$ $m \cdot \vec{g}$ (HIBBELER, 2011), então, obtêm-se a equação que determina a força horizontal real (equação 7):

$$
\begin{aligned}
& F_{h R}-\mu \cdot m \cdot g=m \cdot a \\
& F_{h R}=m \cdot(g \cdot \mu+a)
\end{aligned}
$$

Onde:

$F_{h R}=$ Força horizontal real (N); $\left(m / s^{2}\right)$

$\mu=$ Coeficiente de atrito (o valor dessa grandeza é encontrado como referência em tabelas e depende dos materiais envolvidos na transmissão).

A força tangencial vertical real também é definida a partir da segunda lei de Newton (equação 6), mas depende apenas da força peso (W), conforme ilustrado na figura 5 .

$$
\begin{gathered}
\sum \vec{F}=m \cdot a \\
F_{v R}-W=m \cdot a
\end{gathered}
$$

Como a força peso é dada por $W=$ $m \cdot g$, então, chega-se a equação que determina a força vertical real (equação 8):

$$
\begin{aligned}
& F_{v R}-m \cdot g=m \cdot a \\
& F_{v R}=m \cdot(g+a)
\end{aligned}
$$

Onde:

$F_{v R}=$ Força vertical real $(\mathrm{N})$;

No primeiro instante são calculadas as forças tangenciais reais e, logo depois, devem ser realizados os cálculos para conhecer a força tangencial corrigida, sendo que para isso deve-se adotar um fator de segurança, o qual oscila entre 1,5 e 3. Esse fator está relacionado em função do tempo que o equipamento é utilizado e o tipo de carga que o mesmo está submetido, conforme tabela 3 .

Tabela 3: Coeficiente de segurança para determinação da força tangencial corrigida.

\begin{tabular}{|l|c|c|c|}
\hline \multirow{2}{*}{\multicolumn{1}{|c|}{ TEMPO DE TRABALHO }} & \multicolumn{3}{|c|}{ TIPO DE CARGA } \\
\cline { 2 - 4 } & Uniforme & Moderada & Severa \\
\hline Ocasional (1/2 hora) & 1,5 & 1,8 & 2,3 \\
Intermediário (3 horas) & 1,8 & 2 & 2,5 \\
$\mathbf{8}$ a 10 horas & 2 & 2,25 & 3 \\
$\mathbf{2 4}$ horas & 2 & 2,5 & 3 \\
\hline
\end{tabular}

Fonte: Catálogo da Koom

Selecionado o coeficiente de segurança adequado, agora é só multiplicá-lo pelos valores das forças reais e, assim, obtêm-se as forças tangenciais corrigidas vertical (equação 9) e horizontal (equação 10).

$$
\begin{aligned}
& F_{v c}=F_{v t} \cdot K \\
& F_{h c}=F_{h t} \cdot K
\end{aligned}
$$

Onde: $(\mathrm{N})$;

$$
F_{v c}=\text { Força tangencial vertical corrigida }
$$

$F_{h c}=$ Força tangencial horizontal corrigida $(\mathrm{N})$;

$\mathrm{K}=$ Coeficiente de Segurança. 
Tendo os valores das forças corrigidas e da velocidade é possível escolher o módulo adequado conforme figuras 8, 9 e 10, lembrando que está sendo considerada a condição ideal de trabalho, ou seja, sem deformações estruturais, colisões, alinhamento perfeito, resumindo, uma cremalheira sem erros de usinagem. A análise é feita cruzando a força tangencial (eixo $\mathrm{x}$ ) com a velocidade linear (eixo y), sendo que o ponto de intersecção marca o módulo de transmissão. Quando o ponto de intersecção estiver entre dois módulos, é conveniente escolher o maior, pois assim não haverá o risco de selecionar um módulo cuja força tangencial seja abaixo do calculado.

Figura 8: Módulo em função da velocidade e da força tangencial para o aço SAE 1045 temperado.

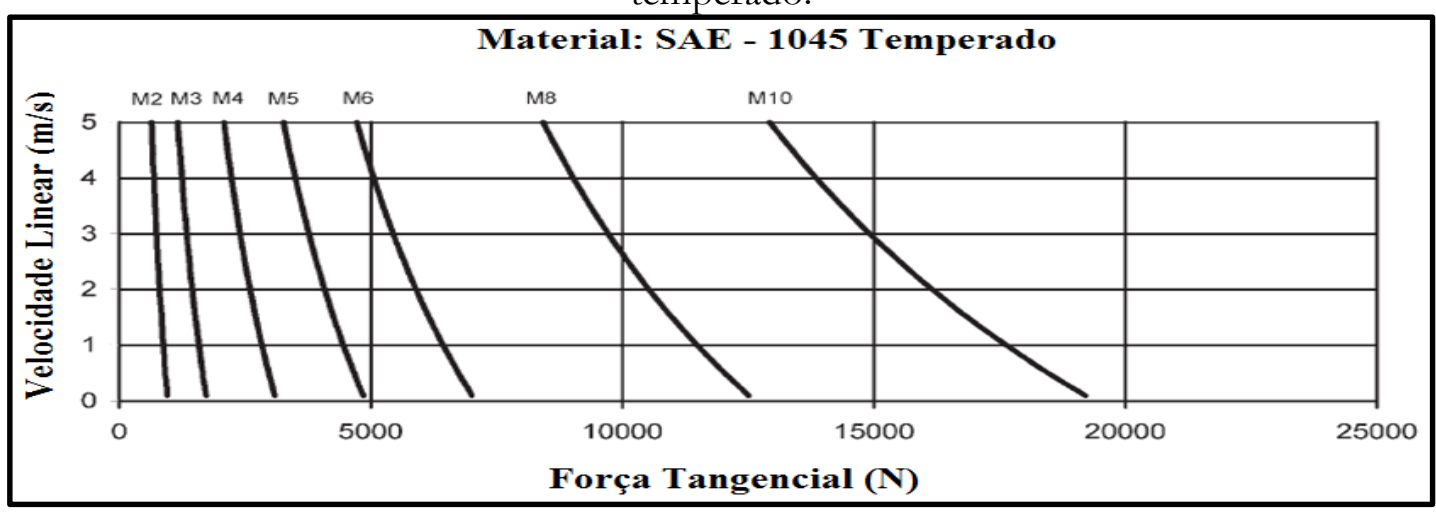

Fonte: Catálogo da Koom

Figura 9: Módulo em função da velocidade e da força tangencial para o aço SAE 1045 com os dentes temperados por indução (HRC>52).

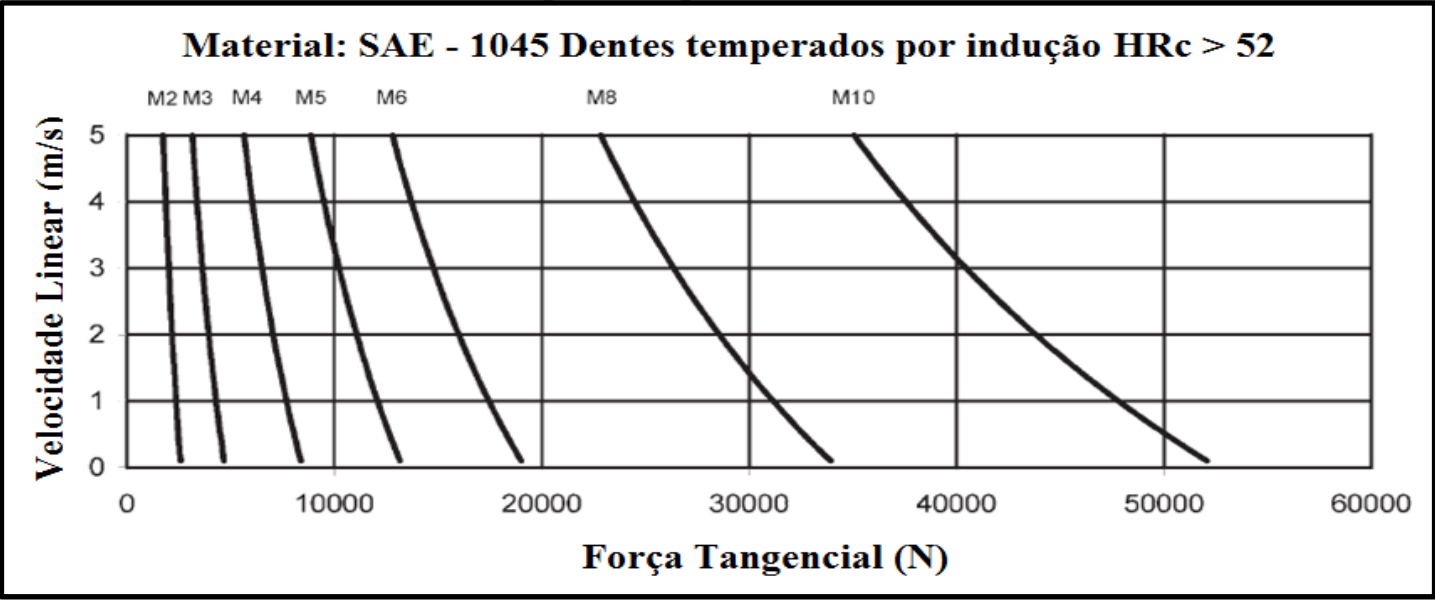

Fonte: Catálogo da Koom 
Figura 10: Módulo em função da velocidade e da força tangencial para o aço SAE 4320/4320 H com dentes cementados e temperados (HRC de 52 a 58).

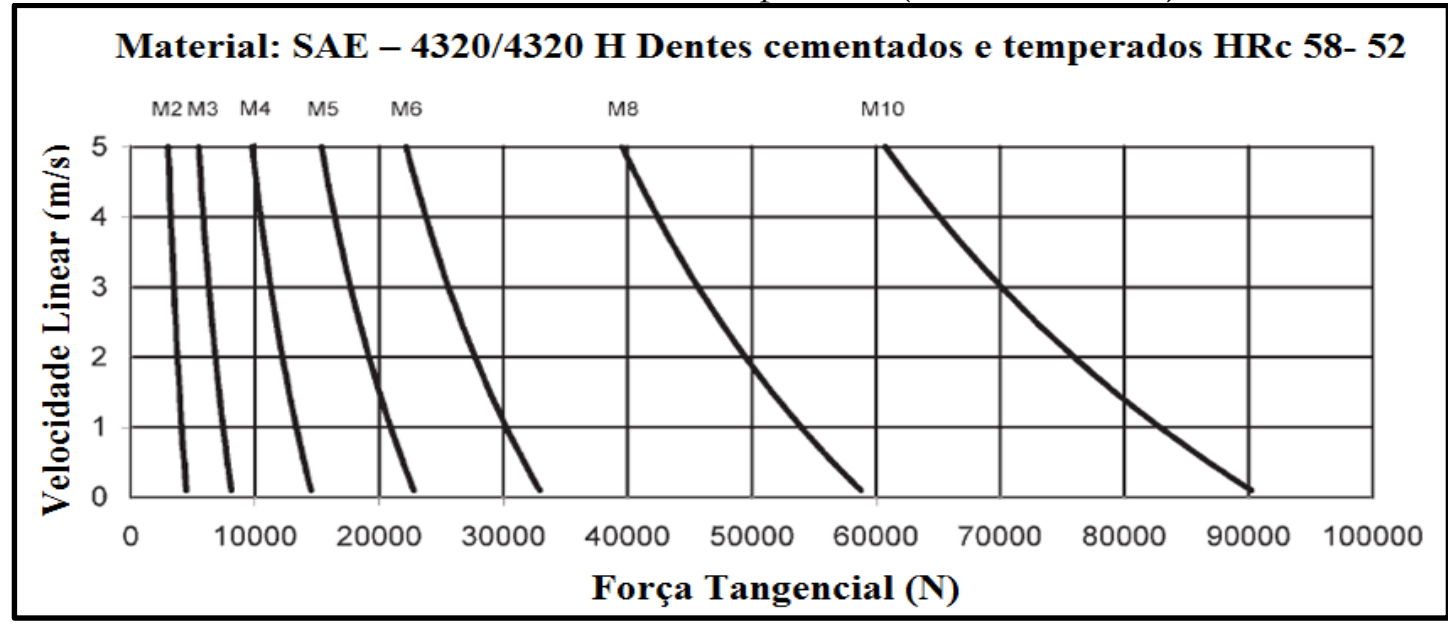

Fonte: Catálogo da Koom

Com o valor do módulo já determinado, basta consultar a tabela 1 e selecionar o número de dentes que o pinhão deve possuir e consequentemente o valor da distância do centro do pinhão à base da cremalheira. Observe que existem de cinco a sete valores do número de dentes para cada módulo, mas procure selecionar um valor intermediário, pois assim, em caso de redimensionamento, existirá a possibilidade de aumentar ou diminuir o número de dentes sem que seja alterado o módulo. A largura da cremalheira deve possuir, no mínimo, a mesma largura que será definida no dimensionamento do pinhão através da análise do critério de desgaste e também da resistência à flexão no pé do dente.

\section{Dimensionamento do pinhão de dentes retos}

Para iniciar o dimensionamento do pinhão, deve-se ter conhecimento de alguns valores iniciais, os quais são simples de se determinar através dos resultados já obtidos no dimensionamento da cremalheira.
Para obter o diâmetro primitivo do pinhão é só utilizar a equação 2 , pois agora já se tem os valores do módulo e do número de dentes.

Segundo Melconian (2008), o torque (MT) é definido como o produto da força tangencial $(\mathrm{F})$ pelo raio $(\mathrm{R})$, como o raio é metade do diâmetro primitivo (d) e sabendo que existe uma perda de rendimento $(\eta)$ na transmissão pinhão cremalheira, então o torque pode ser obtido através da equação 11.

$$
M_{T}=\frac{d . F}{2 . \eta}
$$

A potência mecânica $(\mathrm{P})$ para fazer com que o mecanismo funcione corretamente pode ser determinada através da equação 12 , onde também foi considerada a perda de rendimento na transmissão.

$$
P=\frac{F \cdot v}{\eta}
$$
13.

Já a rotação (n) é calculada pela equação

$$
n=\frac{30 . P}{\pi \cdot M_{T}}
$$


Por já se ter os valores do diâmetro primitivo, do módulo e do número de dentes, o critério de desgaste fica resumido, pois falta apenas determinar a largura do pinhão, a qual será a mesma para a cremalheira, através da equação 14 (MELCONIAN, 2008).

$$
y=\frac{b}{d}
$$

Onde:

$b=$ Largura do pinhão/cremalheira;

$d=$ Diâmetro primitivo do pinhão.

O valor de y, segundo Melconian (2008), depende de como o pinhão estará apoiado, para engrenagem bi apoiada o valor de y deve ser $\leq 1,2$ e para engrenagem em balanço o valor de y deve ser $\leq 0,75$.

Resta agora analisar o critério de resistência à flexão no pé do dente para verificar se as dimensões do pinhão não estão nem subdimensionadas e nem superdimensionadas. Para isso deve-se calcular a tensão máxima no pé do dente expressa pela equação 15 e comparar o resultado com a tensão admissível do material utilizado.

$$
\sigma_{m a ́ x}=\frac{F . q . \varphi}{b . m} \ll \bar{\sigma} \text { mat }
$$

Onde: do dente;

$\sigma_{m a ́ x}=$ Tensão máxima de flexão no pé

$$
\bar{\sigma} \text { mat }=\text { Tensão admissível do material; }
$$

$q$ = Fator de forma (é em função do número de dentes e pode ser encontrado em tabelas nos livros de elementos de máquinas);

$\varphi=$ Fator de serviço (é em função do tipo de aplicação e do tempo de utilização diário e pode ser encontrado em tabelas nos livros de elementos de máquinas).

Se a tensão máxima de flexão no pé do dente for menor que a tensão admissível do material, o pinhão estará aprovado. Caso o resultado seja maior que a tensão admissível, o pinhão deverá ser redimensionado. As grandezas que podem ser redimensionadas são a largura e o módulo. Recomenda-se, se possível, redimensionar a largura, pois não irá interferir em outras grandezas, é só recalcular através da equação 16 (que nada mais é do que a equação 15 modificada) e depois testar novamente a razão b/d, conforme equação 14.

$$
b=\frac{F \cdot q \cdot \varphi}{\bar{\sigma}_{\text {mat }} \cdot m}
$$

Caso não seja possível redimensionar a largura por limitações do projeto, então se deve redimensionar o módulo através da equação 17, mas alterando-se o módulo, automaticamente deverá ser alterado o diâmetro primitivo e consequentemente irá modificar os valores do torque, potência e rotação e também deverá testar novamente a razão b/d, conforme equação 14 .

$$
m=\frac{F \cdot q \cdot \varphi}{\bar{\sigma}_{\text {mat }} \cdot b}
$$

Um fator muito importante para o conjunto pinhão/cremalheira diz respeito ao acabamento da superfície dos dentes e para que isso seja possível devem ser seguidos alguns parâmetros durante a construção desse elemento mecânico.

Conforme Budynas (2005, p. 642) além das velocidades e forças que são submetidas às engrenagens, ainda podem existir interferências de forças dinâmicas adicionais, caso hajam erros nos perfis dentados. No entanto, esses defeitos podem ser corrigidos após o corte, seja pelo processo de rebarbação ou mesmo por brunimento. O processo de retífica e lapidação é utilizado para dentes de engrenagens já endurecidos após tratamento térmico, sendo que a operação de retífica gera dentes com boa precisão, enquanto que no processo da lapidação os dentes da engrenagem e da lapidadora deslizam de forma axial, 
propiciando que a parte superficial se desgaste de maneira igual.

O catálogo da Koom (s.d.) indica que o acabamento superficial utilizado nos dentes do conjunto pinhão/cremalheira seja de 0,8 Ra.

\section{Conclusão}

Neste artigo foi demonstrado como deve ser o procedimento para se dimensionar cremalheiras de dentes retos, indicando todos os cálculos e baseando-se em dados de uma empresa espanhola (KOOM) fabricante de sistemas de transmissão que disponibilizou algumas tabelas e gráficos para obtenção do módulo e a seleção do material adequado para a fabricação do sistema pinhão/cremalheira, os quais já foram testados na prática. Foi elaborada uma sequência para esse dimensionamento de uma forma simples e objetiva, procurando sempre descrever a maneira correta de realização dos cálculos e a interpretação de tabelas e gráficos.

Com o objetivo de entender na prática o funcionamento do sistema pinhão/cremalheira, foram analisados alguns equipamentos e/ou máquinas em uma indústria mecânica (Metalúrgica Mecano Fabril) e até foram obtidas algumas fotografias e disponibilizadas nesse artigo. Além disso, também para melhorar a compreensão da aplicação do conjunto pinhão cremalheira, foi realizado o uso de imagens e desenhos técnicos.

Vale destacar que o artigo abordou apenas o dimensionamento do conjunto dentado de dentes retos, mas que para a cremalheira existe também a opção de se trabalhar com dentes helicoidais.

\section{Referências Bibliográficas}

1. BUDYNAS, Richard G., MISCHKE, Charles R., SHIGLEY, Joseph E. Projeto de

Engenharia Mecânica, $7^{\mathrm{a}}$ edição. Porto Alegre: Bookman, 2005.

2. CLUBE DA ELETRÔNICA; Transmissão e transformação de movimento; Disponível em: $<$ http://www.clubedaeletronica.com.br/Me canica/Universo $\% 20$ mecanica $\% 20$ TC2000/univ3c.pdf $>$ Acesso em 27 Dezembro 2013.

3. EDUCAÇÃO TECNOLÓGICA; Engrenagens - Conceitos básicos;

Disponível em:<http://2000pt.net/educa caotecnologica/engrenagens.pdf $>$ Acesso em 20 Janeiro 2014.

4. HALLIDAY. David; RESNICK. Robert. Fundamentos de física. 9 ed. Rio de Janeiro: LTC, 2012. 88p

5. HIBBELER, Russell C. Dinâmica: mecânica para engenharia.12 ed. São Paulo: Pearson Prentice Hall, 2011. 504p

6. IEFP, Transformação de movimento circular em movimento de translação retilíneo contínuo; Disponível em: $<$ http://elearning .iefp.pt/pluginfile.php/47218/mod_resourc e/content/0/CDrom/Estudo/Mecanica/D _Transmiss_o_de_Movimento/frame_12.ht m> Acesso em 27 Dezembro 2013. 
7. KOOM Transmission de Precición; 2007;

Catàlogo delas transmisiones por cremallera Piñón. Disponível em:<http://www.mecapedia.uji.es/catalogo s/engranaje/spitkoom.1.pdf $>$ Acesso em 28Dezembro 2013.

8. MELCONIAN, Sarkis. Elementos de máquinas. 9. ed. rev. São Paulo: Érica, 2008.

9. RLG - Indústria e Equipamentos de Segurança Ltda.; Movimentador deslizante de cremalheira; Disponível em: <http://www.rlg.ind.br/horizontal.html> Acesso em 29 Janeiro 2013.

10. SARDÁ, Prof. Alexandre Augusto Pescador; Engrenagens; Disponível em: <ftp://ftp.demec.ufpr.br/disciplinas/TM12 9/Prof.\%20Pescador/Engrenagens1.pdf> Acesso em 27 Dezembro 2013.
11. SHIGLEY, Joseph Edward, Tradução de CARVALHO, Edival Ponciano de. Elementos de máquinas. Rio de Janeiro: LTC - Livros Técnicos e Científicos Editora S.A. 1984.

12. THYSSEN KRUPP MATERIALS IBÉRICA; Aceros de Construcción; Disponível em: <http://www.thyssenkrupp materials-iberica.es/htm/pdf_aconstruccion. pdf $>$ Acesso 27 Janeiro 2014.

13. WMH Herion; Partofyour drive;

Disponível em: <http://www.apexdyna brasil.com.br/images/content/pdf/definico es_tecnicas/Rodas $\% 20$ dentadas $\% 20$ $\% 20 \mathrm{WMH} \% 20$ Herion.pdf> Acesso em 06 Janeiro 2014. 